\title{
Integrated omics data of two annual ryegrass (Lolium multiflorum L.) genotypes reveals core metabolic processes under drought stress
}

Ling Pan ${ }^{1}$, Chen Meng ${ }^{2}$, Jianping Wang ${ }^{3}$, Xiao Ma ${ }^{1}$, Xiaomei Fan ${ }^{4}$, Zhongfu Yang ${ }^{1}$, Meiliang Zhou ${ }^{5^{*}}$ and Xinquan Zhang ${ }^{1 *}$

\begin{abstract}
Background: Annual ryegrass (Lolium multiflorum L.) is a commercially important, widely distributed forage crop that is used in the production of hay and silage worldwide. Drought has been a severe environmental constraint in its production. Nevertheless, only a handful of studies have examined the impact of short-term drought stress on annual ryegrass. The aim of this study was to explore how stress-induced core metabolic processes enhance drought tolerance, or adaptation to drought, in annual ryegrass.

Results: We profiled the transcriptomes, proteomes, and metabolomes of two annual ryegrass genotypes: the drought-resistant genotype "Abundant 10" and drought-susceptible genotype "Adrenalin 11." We identified differentially expressed metabolites and their corresponding proteins and transcripts that are involved in 23 core metabolic processes, in response to short-term drought stress. Protein-gene-metabolite correlation networks were built to reveal the relationships between the expression of transcripts, proteins, and metabolites in drought-resistant annual ryegrass. Furthermore, integrated metabolic pathways were used to observe changes in enzymes corresponding with levels of amino acids, lipids, carbohydrate conjugates, nucleosides, alkaloids and their derivatives, and pyridines and their derivatives. The resulting omics data underscored the significance of 23 core metabolic processes on the enhancement of drought tolerance or adaptation to drought in annual ryegrass.

Conclusions: The regulatory networks were inferred using MCOA and correlation analysis to reveal the relationships among the expression of transcripts, proteins, and metabolites that highlight the corresponding elements of these core metabolic pathways. Our results provide valuable insight into the molecular mechanisms of drought resistance, and represent a promising strategy toward the improvement of drought tolerance in annual ryegrass.
\end{abstract}

Keywords: Metabolism, Drought, Annual ryegrass (Lolium multiflorum), Omics, Transcriptome, Proteome, Metabolome

\section{Background}

Drought is a severe environmental constraint to seed germination, plant growth, and productivity $[1,2]$. Plants employ physiological and molecular mechanisms of drought tolerance to cope with water shortages. Indeed, the drought-response mechanisms developed by plants at the

\footnotetext{
*Correspondence: zhoumeiliang@caas.cn; zhangxq@sicau.edu.cn ${ }^{5}$ Institute of Crop Sciences, Chinese Academy of Agricultural Sciences, Beijing, China

'Department of Grassland Science, College of Animal Science and Technology, Sichuan Agricultural University, Chengdu, China

Full list of author information is available at the end of the article
}

cellular level are essential, as they allow tolerance in plants that facilitates cellular homeostasis [3]. Mounting evidence has confirmed that plant species that are more tolerant to drought stress maintain higher levels of unsaturated fatty acids, such as hexadecenoic acid, palmitic acid, pimelic acid, stearic acid, and linolenic acid, all of which intervene in cases of compromised membrane fluidity and cellular functions [4-6]. In addition, the synthesis of amino acids, such as valine, leucine, phenylalanine, proline, and histidine, can contribute to turgor maintenance through osmotic adjustment when plants, 
especially resistant cultivars, are subjected to gradually increasing drought stress [7].

Plants exhibiting high drought tolerance are promising candidates for studies in drought-related genes, proteins, and metabolites [8]. Transcriptomic, proteomic, and metabolomic profiling are highly useful approaches to dissecting the complex networks of regulatory mechanisms at multiple levels in plants $[9,10]$. In addition, multiple co-inertia analysis (MCoA) is a critical method for integrating data from several multi-omics datasets [11]. One of the advantages of MCoA is that it can be used to analyze a subset of variables (e.g., transcripts, proteins, and metabolites) that are present in two or more datasets. Several recent studies using comparative physiological, metabolomic, and transcriptomic analyses have provided deep insights into the mechanisms associated with improving abiotic stress resistance through the application of exogenous melatonin [12]. Similarly, combined analyses of the transcripts, proteome, and metabolites have been used to understand the manner in which core metabolic processes enhance carotenoid synthesis in transgenic maize [13]. "Omics" studies have also been performed, using large drought datasets for forage grasses with differing levels of sensitivity, both under water stress and non-stress conditions [14, 15].

Annual ryegrass (Lolium multiflorum L.), a species closely related to perennial ryegrass (Lolium perenne), is a commercially important forage crop that is widely cultivated for the production of hay and silage worldwide [16], including southern China. In recent years, severe short-term (daily to monthly) droughts have occurred frequently over southern China, causing a severe adverse effect on grass productivity $[17,18]$. As such, the economic importance of annual ryegrass has encouraged many researchers to study the physiological and molecular bases of drought tolerance. Nevertheless, only a handful of studies have examined the impact of shortterm drought stress on annual ryegrass, using an omics approach [19].

In our recently published study, we developed two annual ryegrass varieties, Abundant 10 and Adrenalin 11, with differing degrees of drought tolerance [20]. In order to explore the molecular mechanism of drought tolerance in these two annual ryegrass genotypes, we identified differentially expressed metabolites and their corresponding proteins and transcripts that are involved in 23 core metabolic processes under short-term drought treatment. The associated regulatory networks were inferred using MCoA and correlation analysis, to reveal the relationships among the expression of transcripts, proteins, and metabolites that highlight the corresponding elements of these core metabolic pathways. This study provides valuable insight into the molecular mechanisms of drought resistance and represents a promising approach toward the improvement of drought tolerance in annual ryegrass.

\section{Methods \\ Plant samples and drought treatments}

Two L. multiflorum genotypes, drought-resistant "Abundant 10" and drought-susceptible "Adrenalin 11," were used in this study. Seedlings were transplanted 7 days after germination into Hoagland's nutrient solution. All seedlings were grown in temperature-controlled growth chambers with 16 -h photoperiods $\left(25^{\circ} / 18{ }^{\circ} \mathrm{C}\right.$ day/night temperature) and relative humidity of $60 \%$. At day 20 , after germination, the seedlings were divided into four groups to be used as the control $(0 \mathrm{~h})$ and various drought-treated samples. The drought-treated samples were placed on plastic trays and naturally air-dried for 1 , 2, and $24 \mathrm{~h}$, respectively (Additional file 1 Figure S1). Ten individual plants from each treatment group were considered a biological replicate. After treatment, three biological replicates were used for total RNA and protein extraction, and six biological replicates were then subjected to gas chromatography-mass spectrometry- (GCMS)-based metabolite identification.

Relative water content (RWC) was calculated using the formula described by Barrs and Kozlowski [21]. The chlorophyll content was calculated according to the method described by Lakra et al. [22]. Relative electrical conductivity (REC) was calculated as the ratio of the initial electrical conductivity (EC) to the final EC [23]. The changes and activity of malondialdehyde (MDA) concentration, superoxide dismutase (SOD), catalase (CAT), and ascorbic acid peroxidase (APX) were each assayed with MDA, SOD, CAT, and APX assay kits, respectively (Comin Biotechnology Co. Ltd., Suzhou, China). A oneway ANOVA was performed using the SPSS Statistics 20.0 software (IBM Corp, Armonk, NY, USA), and means were compared using the least significant difference (LSD) test to determine significant differences $(P<$ $0.05)$.

\section{Transcriptome sequencing and analysis}

Total RNA was isolated from samples, using the TRIzol reagent (Agilent Technologies, Santa Clara, USA). A mass of $5 \mu \mathrm{g}$ per sample was collected for cDNA library construction, using the NEB Next ${ }^{\circ}$ Ultra $^{\mathrm{m}}$ Directional RNA Library Prep Kit for Illumina ${ }^{\circ}$ (NEB, USA). The cDNA Library was validated by two different methods to determine the average molecular length: 1) the Agilent 2100 Bioanalyzer (Agilent DNA 1000 Reagents; Agilent Technologies) and 2) real-time quantitative PCR (QPCR; TaqMan Probe; Thermo Fisher Scientific, Waltham, MA, USA). The qualified libraries (average length of fragment was between 250 and $350 \mathrm{bp}$ ) were amplified on the cBot System to generate the cluster on the flow 
cell using the TruSeq PE Cluster Kit V3-cBot-HS (Illumina, San Diego, CA, USA). The amplified flow cell was subjected to paired-end sequencing using the HiSeq 2000 sequencing system (Illumina, USA). As a reference genome had not been previously created, the clean reads were assembled as the reference genome via the Trinity software (https://github.com/trinityrnaseq/trinityrnaseq/ wiki) [24]. Read counts per gene were expressed as the expected number of fragments per kilobase of transcript sequence per million base pairs sequenced (FPKM). The $P$-values were adjusted using the Benjamini and Hochberg's approach to control the false discovery rate (FDR). Gene Ontology (GO) enrichment analysis of differentially expressed genes (DEGs) was implemented with the GOseq $\mathrm{R}$ software package, in which the gene length bias was corrected. The GO terms with DEGs (FDR $\leq$ 0.001 and a fold change $\geq 2$ ) were used for functional enrichment analysis. Genes with an adjusted $P$-value below 0.05 , as determined by the DESeq software, were assigned as differentially expressed, and employed in the GO and Kyoto Encyclopedia of Genes and Genomes (KEGG) analyses. The KEGG enrichment analysis of DEGs in the KEGG database (http://www.genome.jp/ kegg/) and the KOBAS (KEGG Orthology Based Annotation System) software $[25,26]$ were used to test the statistically significant enrichment of DEGs in KEGG pathways.

\section{Protein identification and data analysis}

Proteins were extracted from samples at four time points throughout the drought stress treatments: $0,1,2$, and $24 \mathrm{~h}$. The extractions were performed with Lysis Buffer 3 containing $1 \mathrm{mM}$ phenylmethylsulfonyl fluoride (PMSF) and $2 \mathrm{mM}$ ethylenediaminetetraacetic acid (EDTA), and suspended at $200 \mathrm{~W}$ for $15 \mathrm{~min}$. Proteins were isolated by centrifugation at $30000 \mathrm{~g}$ for $15 \mathrm{~min}$ at $4{ }^{\circ} \mathrm{C}$, after which $5 \times$ volume of chilled acetone and $10 \%$ $(v / v)$ trichloroacetic acid were added at $-20{ }^{\circ} \mathrm{C}$.

After two rounds of centrifugation, the supernatant was carefully discarded and the precipitate was washed three times with cold acetone. The protein pellet was air-dried by lyophilization and dissolved in Lysis Buffer (7 M urea, $2 \mathrm{M}$ thiourea, 4\% NP40, $20 \mathrm{mM}$ Tris- $\mathrm{HCl}$, $\mathrm{pH}$ 8.0-8.5). The protein pellet was then suspended for $15 \mathrm{~min}$ and centrifuged at $4{ }^{\circ} \mathrm{C}$ at $25000 \mathrm{~g}$ for $15 \mathrm{~min}$, and the supernatant was collected.

To reduce the number of disulfide bonds in the proteins of the supernatant, $10 \mathrm{mM}$ dithiothreitol (DTT) was added, and the mixture was left for $1 \mathrm{~h}$ at $56{ }^{\circ} \mathrm{C}$. Subsequently, $55 \mathrm{mM}$ iodoacetamide (IAM) was added to block the cysteines, after which samples were kept in a dark room for $1 \mathrm{~h}$. The supernatant of the proteins was kept at $-80{ }^{\circ} \mathrm{C}$. Protein samples of $100 \mu$ g each were added to $2.5 \mu \mathrm{g}$ Trypsin (Promega, Madison, WI, USA) with a weight ratio of 40 protein: 1 trypsin, and kept at $37{ }^{\circ} \mathrm{C}$ for $4 \mathrm{~h}$. The peptides were vacuum-dried using Strata-X, and reconstituted in $0.5 \mathrm{M}$ triethylammonium bicarbonate (TEAB) based on the manufacturer's protocol for the 8-plex iTRAQ reagent (Applied Biosystems, USA). This solution contained one unit of thawed and reconstituted iTRAQ reagent in $24 \mu \mathrm{L}$ isopropanol. The peptides were labeled with isobaric tags, pooled, and then vacuum-dried.

Each fraction was re-suspended in buffer A (2\% acetonitrile $[\mathrm{ACN}], 0.1 \%$ formic acid $[\mathrm{FA}]$ ) and centrifuged at $20000 \mathrm{~g}$ for $10 \mathrm{~min}$. The final concentration of peptides was on average approximately $0.5 \mu \mathrm{g} / \mu \mathrm{L}$. The supernatant $(10 \mu \mathrm{L})$ was evaluated using a LC-20 AD NanoHPLC pump (Shimadzu, Kyoto, Japan) with an autosampler, and peptides were eluted onto an analytical C18 column (inner diameter $75 \mu \mathrm{m}$ and column length $15 \mathrm{~cm}$ ). The samples were loaded over $4 \mathrm{~min}$, and the solvent gradient was run from $5 \%$ buffer B $(96 \% \mathrm{ACN}$, $0.1 \% \mathrm{FA}$ ) for $0-8 \mathrm{~min}$, with a linear gradient to $35 \%$ buffer B for 8-43 min, maintained at $60 \%$ buffer B for $43-$ $48 \mathrm{~min}$, and returned to $5 \%$ buffer B for 55-65 min.

Data acquisition was performed using a Triple TOF 5600 System (SCIEX, Framingham, MA, USA) fitted with a Nanospray III source (SCIEX) and a pulled quartz tip as the emitter (New Objectives, Woburn, MA, USA). Data were acquired using an ion spray with a $2.5 \mathrm{kV}$ voltage. The curtain and nebulizer gases were set at $30 \mathrm{psi}$ and $15 \mathrm{psi}$, respectively, and the interface heater temperature was $150{ }^{\circ} \mathrm{C}$. The mass spectrometer was operated with a resolving power (RP) of 30,000 FWHM (full width at half maxima) for time-of-flight mass spectrometry (TOF/MS) scans. Survey scans were obtained in $250 \mathrm{~ms}$ and up to 30 product ion scans were acquired (cut-off threshold was 120 counts/s). Raw data files were transformed into Mascot generic format (MGF) files using the Proteome Discoverer software.

Raw data files acquired from the Orbitrap analyzer were converted into MGF files using the Proteome Discoverer 1.2 software (Thermo Fisher). The Mascot 2.3.02 search engine (Matrix Science, London, UK) was used to identify and quantify proteins. An automatic decoy database search was performed in Mascot, by choosing the decoy checkbox in which a random sequence database was generated and tested for raw spectra. The real database was also tested for raw spectra. Only peptides with a 95\% confidence interval from the Mascot probability analysis were counted as identified. The identification of each protein involved at least one unique peptide. The quantitative protein ratios were weighted and normalized using the median ratio determined by Mascot. We considered only data with values of $P<0.05$ and fold changes $>1.2$ as significant. Functional annotations of identified proteins were performed using the Blast2GO 
program against the non-redundant (NR) protein database. The KEGG and clusters of orthologous groups (COG) databases were applied to classify the identified proteins.

\section{Metabolome analysis}

Six biological replicates of each sample were analyzed for non-treated and drought-treated seedlings (drought for $24 \mathrm{~h}$ ) in the two L. multiflorum genotypes. Approximately $50 \mathrm{mg}$ of powdered samples were extracted in $1 \mathrm{~mL}$ of an $80 \%$ methanol, $20 \%$ distilled water solution for $30 \mathrm{~min}$ at $4{ }^{\circ} \mathrm{C}$. During the extraction process, the samples were centrifuged for $10 \mathrm{~min}$ at $12,000 \mathrm{~g}$. The supernatant was removed and the pellet was subjected to further extraction in $60 \%$ methanol, and then in water at $4{ }^{\circ} \mathrm{C}$, as described above. Metabolite profiling was performed on an Agilent 1290 Infinity Liquid Chromatography System (Agilent Technologies) equipped with a $2.1 \mathrm{~mm} \times 100 \mathrm{~mm}$ C18 reverse-phase column, with a 1.8- $\mu \mathrm{m}$ particle size (Waters Corp., Milford, MA, USA). The column was maintained at $40{ }^{\circ} \mathrm{C}$ and the injected sample volume was $4 \mu \mathrm{L}$. Mass spectrometry (MS) experiments were performed on an Agilent 6530 AccurateMass Q-TOF/MS (Agilent Technologies) equipped with an electrospray ionization source. The binary gradient had a flow rate of $0.35 \mathrm{~mL} / \mathrm{min}$, and a gradient elution from $5 \%$ to $95 \%$ acetonitrile separated all compounds. The total run time was $34 \mathrm{~min}$.

The electrospray source parameters were optimized as follows: positive mode- sampling cone $35 \mathrm{kV}$, capillary voltage $4 \mathrm{kV}$, extraction cone $3 \mathrm{~V}$, source temperature $100{ }^{\circ} \mathrm{C}$, desolvation temperature $350{ }^{\circ} \mathrm{C}$, cone gas flow $50 \mathrm{~L} / \mathrm{h}$, and desolvation gas flow $600 \mathrm{~L} / \mathrm{h}$. Negative mode- sampling cone $50 \mathrm{kV}$, capillary voltage $3.5 \mathrm{kV}$, extraction cone $4 \mathrm{~V}$, source temperature $100{ }^{\circ} \mathrm{C}$, desolvation temperature $300{ }^{\circ} \mathrm{C}$, cone gas flow $50 \mathrm{~L} / \mathrm{h}$, and desolvation gas flow $700 \mathrm{~L} / \mathrm{h}$. For accurate mass acquisition, a lock mass of leucine enkephalin (Lock mass) at a concentration of $0.2 \mathrm{ng} / \mathrm{mL}$ was used via a lock spray interface for the positive ion mode $([\mathrm{M}+\mathrm{H}]+=$ $556.2771 \mathrm{Da})$ and negative ion mode $([\mathrm{M}-\mathrm{H}]-=$ $554.2615 \mathrm{Da}$ ) to ensure accuracy during the MS analysis.

After filtration, a peak table was created that included information on the retention time (RT), mass, and ion intensity of all identified components. Raw data were first preprocessed with a Mass Profiler (Agilent Technologies) and input to the Simca-P 13.0 program for multivariate analysis (i.e., principal component analyses $[\mathrm{PCA}]$ and partial least squares discriminant analysis [PLS-DA]). Data were expressed as the mean \pm SD. Variable importance in the projection (VIP) values were obtained for variables in the orthogonal projections to latent structures discriminant analysis (OPLS-DA) model in order to select the differentially expressed metabolites. An independent $t$-test $(P<0.05)$ was used to determine whether the individual metabolites (candidate biomarkers) obtained from PLS-DA modeling, exhibited statistically significant differences between groups at the univariate analysis level. Only the metabolites with a VIP $>1$ and a $P$-value $<0.05$ were defined as differential metabolites. The MS analysis system was used to identify the characteristic metabolites corresponding to the featured peak in the Metlin database (http://metlin.scripps.edu) [27]. The KEGG database was also used to link differential metabolite levels to functional metabolic pathways in the drought-treated groups, compared to those in the control groups.

\section{Integrative analysis using multiple co-inertia analysis and gene set enrichment analysis}

The MCoA was performed using the Bioconductor software package omicade4 [28]. For consistency, we selected 1-, 2-, and 24-h treatments as the drought treatment groups, and $0 \mathrm{~h}$ as the control group. The $0-\mathrm{h}$ and 24-h metabolomic profiles were used as the control and drought treatment profiles, respectively. The mean FPKM in the transcriptomic data (three replicates), ratios in the proteomics data (two replicates), and peak intensity in the metabolic data (six replicates) were used in the MCoA. All values were log-transformed. The MCoA can deal with multiple datasets; therefore, we did not merge the positive and negative channels in the metabolite profiling, which resulted in four datasets for each of genotypes. The Gene Set Enrichment Analysis (GSEA) software (version 2.2.2) was used for gene set enrichment analysis [29]. The gene set information was collected from GO and KEGG pathway databases, as in the differential expression analysis. Gene sets containing more than 1000 or fewer than five candidates were excluded. A permutation text with 1000 permutations was used to evaluate the significance of the enrichment results. The default settings for other parameters were used.

\section{Quantitative real-time-PCR analysis and western blot}

Changes in the expression of 26 genes were verified by real-time PCR analysis, and detailed information on these genes is listed in Additional file 2 Table S1. Realtime RT-PCR was performed on the ABI7500 Real-Time PCR System (Applied Biosystems) using the SYBR ${ }^{\circ}$ Premix EX-Taq ${ }^{\text {Th }}$ II kit. Reverse transcription was performed using the Bio-Rad iScript cDNA Synthesis Kit. The PCR amplifications were conducted in a volume of $20 \mu \mathrm{L}$, containing $10 \mu \mathrm{L}$ PCR-mix, $2.5 \mu \mathrm{L}$ of genomic DNA, $5.5 \mu \mathrm{L}$ ddH2O, and $1 \mu \mathrm{L}$ of each primer. The thermocycler was set to touchdown mode according to the following program: $95{ }^{\circ} \mathrm{C}$ for $30 \mathrm{~s}, 1$ cycle; $95{ }^{\circ} \mathrm{C}$ for $5 \mathrm{~s}$, 40 cycles; and $60{ }^{\circ} \mathrm{C}$ for $34 \mathrm{~s}, 1$ cycle. A melting curve 
was generated by heating the sample to $95^{\circ} \mathrm{C}$. Real-time PCR data was then analyzed by the comparative CT method.

An equal amount $(10 \mu \mathrm{g})$ of each protein sample was loaded for sodium dodecyl sulfate polyacrylamide gel electrophoresis (SDS-PAGE) (1.5 mm gel thickness). Migration of proteins in the gel was conducted at $150 \mathrm{~V}$ until the blue band from the sample buffer ran out of the gel. Protein-Marker IV was also loaded to determine the molecular weight of the proteins. Proteins were then transferred onto a polyvinylidene fluoride (PVDF) membrane (Millipore, USA). The following antibodies were used in the western blot analysis: actin (ACT), chitinase $(\mathrm{CHN})$, glutamine synthetase (GS1), methionine synthase (MTR), UDP-glucose pyrophosphorylase (UGPase), glutamate dehydrogenase 1 (GDH1), serine hydroxyl methyltransferase (SHMT), and glutamine synthetase (GlnA) (Agrisera, Sweden). The PVDF membrane was probed with primary antibodies and developed using enhanced chemiluminescence detection (PerkinElmer, Waltham MA, USA). The blots were detected using the BeyoECL Plus (P0018) system. The images were obtained using the ChemiDoc TM MP imaging system, and the quantifications were conducted with the Image Lab TM V5.1 software.

\section{Results}

\section{Drought stress induced growth and physiological changes in L. multiflorum}

To determine the morphological and physiological impact of drought stress on annual ryegrass, both drought-resistant and drought-susceptible genotypes were subjected to sustained drought for 5 weeks (Fig. 1a-b). Differences between the genotypes were observed in seedling height $(\mathrm{SH})$, chlorophyll $(\mathrm{a}+\mathrm{b})$ content, REC, RWC, root-to-shoot ratio (RSR), malondialdehyde (MDA) content, catalase (CAT), superoxide dismutase (SOD), and ascorbic acid peroxidase (APX) activities (Fig. 1c-k). The impact of drought stress on RWC was apparently reduced among treated seedlings after the 14-day drought treatment, and there was a significant difference in response between the two annual ryegrass genotypes (Fig. 1c). The REC and chlorophyll $(\mathrm{a}+\mathrm{b})$ content of the susceptible plants exhibited a dramatic reduction, in comparison to those of the tolerant plants (Fig. 1d-e). Prior to the application of drought stress, MDA content was invariant among the control samples of the two annual ryegrass genotypes. However, susceptible genotype revealed significantly higher MDA contents, relative to those of the tolerant genotype after 14 days of drought stress (Fig. 1f). Higher levels of CAT, SOD, and APX activity were observed among the tolerant genotype exposed to long-term drought (Fig.
$1 g-i)$, whereas no significant changes were noted in $\mathrm{SH}$ when subjected to drought stress (Fig. 1j). However, a highly significant increase in RSR was observed in the tolerant genotype in comparison to the susceptible genotype after annual ryegrass seedlings were treated with drought stress for 21 days (Fig. 1k).

\section{Metabolite profiling of two L. multiflorum genotypes revealed changes in metabolites under drought stress} The differentially expressed metabolites of the two $L$. multiflorum genotypes were identified following exposure to $24 \mathrm{~h}$ of drought stress, using LC-MS and the OPLS-DA model. Different types of compounds were detected in positive and negative modes including: lipids; amino acids; organic acids; carbohydrates and carbohydrate conjugates; nucleosides, nucleotides, and their analogs; indoles and their derivatives; alkaloids and their derivatives; amine compounds, pyridines and their derivatives, among others (Additional file 3: Figure S2). additional(DEMs) exhibited contrasting expression levels between the drought-tolerant and drought-sensitive genotypes under drought stress, particularly lipids, amino acids, organic acids, amine compounds, and pyridines and their derivatives. Significant correlations were noted among the levels of these compounds, according to Spearman two-tailed correlation analyses $(P \leq 0.05$ and $r^{2}>0.65$ or $P \leq 0.05$ and $r^{2}<-0.65$; Additional file 4 : Figure S3). Lipids and amino acids more favorably ionized in positive ionization mode (Additional file 4: Figure S3A), whereas organic acids generated higher signal intensities in positive ionization mode (Additional file 4: Figure S3). We found that lipid levels exhibited the greatest number of significant correlations with other metabolites, followed by amino acids, and organic acids.

Among these compounds, a portion of the DEM scan was mapped onto multiple core metabolic processes, for example, valine, leucine, and isoleucine biosynthesis; valine, leucine, and isoleucine degradation; $\alpha$-linolenic acid metabolism; biotin metabolism; phenylalanine, tyrosine, and tryptophan biosynthesis; tropane, piperidine, and pyridine alkaloid biosynthesis; phenylalanine metabolism; sphingolipid metabolism; fatty acid biosynthesis; phenylpropanoid biosynthesis; glycerolipid metabolism; pantothenate and $\mathrm{CoA}$ biosynthesis; histidine metabolism; tryptophan metabolism; galactose metabolism; purine metabolism; cysteine and methionine metabolism; fatty acid metabolism; arginine and proline metabolism; 2oxocarboxylic acid metabolism; cutin; suberin and wax biosynthesis; biosynthesis of amino acids; and the citrate cycle (TCA cycle; Table 1). 


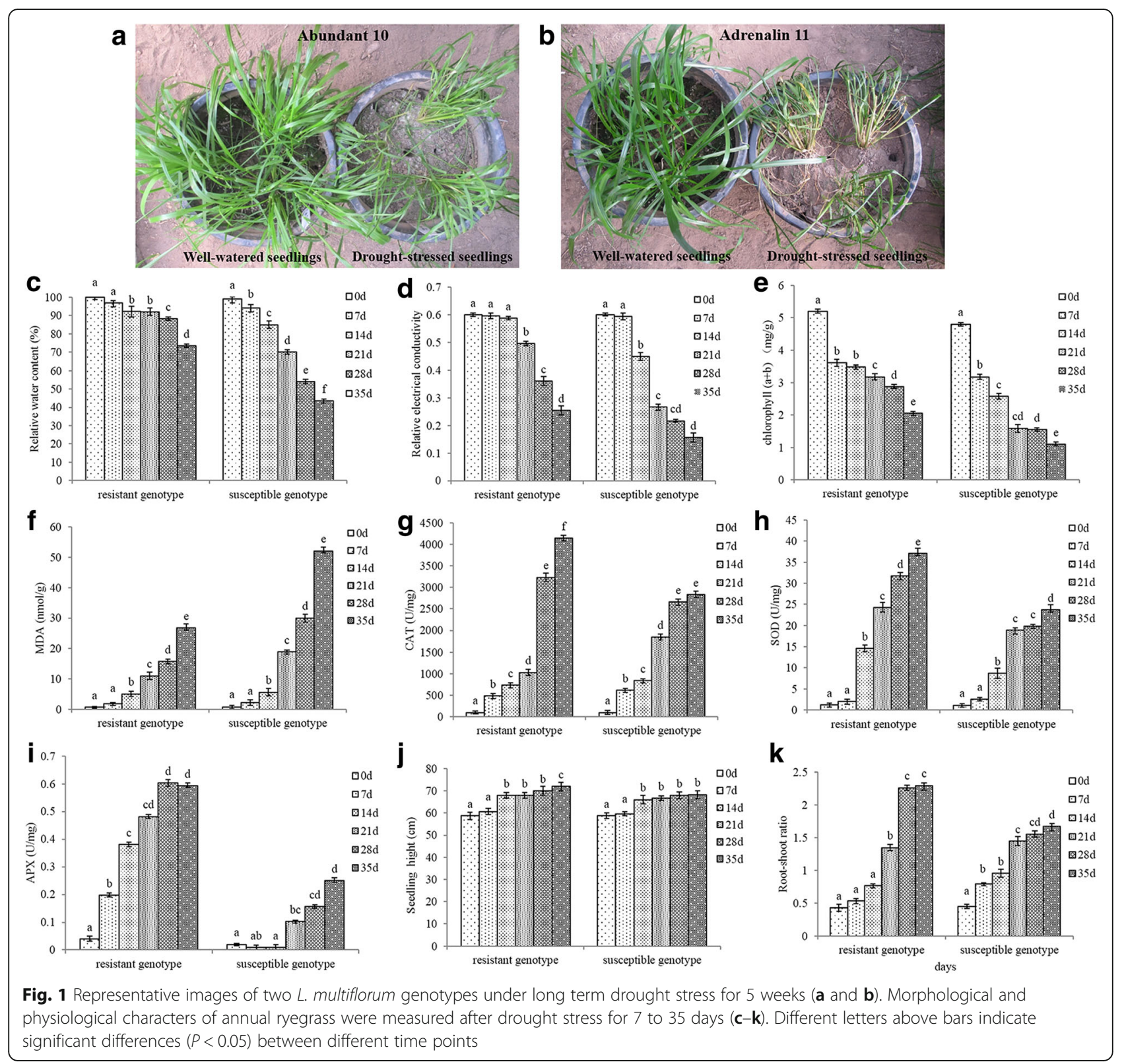

\section{Comparative proteomics and transcriptomic profiling reveals differences in the expression of proteins and genes regulating core metabolism}

To investigate the differentially abundant proteins induced by drought treatment, we performed comparative proteomics analysis on the two annual ryegrass genotypes at four different time points $0,1,2$, and $24 \mathrm{~h}$. A total of 26,189 unique peptides matching 8224 proteins were identified by Mascot with a high level of confidence (all with a 1\% FDR), of which 1395 were differentially abundant between the drought-susceptible and droughtresistant genotypes (Fig. 2a). To understand further the function and features of these proteins, differentially expressed proteins (DEPs) were identified using
Blast2GO and the KEGG database. Most of these proteins were located in organelles, cells, membranes, or nuclei (Fig. 2b). The KEGG pathway enrichment analysis revealed that the abundance of DEPs involved in metabolic pathways that are related to the metabolites identified by LC-MS were dramatically affected by drought (Fig. 2c). This identified the drought-induced proteins associated with stress responses that might contribute to enhanced tolerance or adaptation to drought in annual ryegrass.

To gain insight into gene expression in annual ryegrass across all four time points of the drought treatment, we performed transcriptomic analysis to identify droughtmediated genes. After filtering out contaminated and 
Table 1 Differentially expressed metabolites mapped to KEGG metabolic pathways

\begin{tabular}{|c|c|c|c|c|}
\hline Metabolite Name & HMDB ID & $\begin{array}{l}\text { KEGGCompound } \\
\text { ID }\end{array}$ & $\begin{array}{l}\text { Fold change (sus_24 } \\
\text { vs.res_24) }\end{array}$ & Pathway Name \\
\hline Pheophorbide a & METPA1634 & C18021 & 0.911 & Porphyrin and chlorophyll metabolism \\
\hline Hexahomomethionine & METPA1758 & C17233 & -1.056168721 & 2-Oxocarboxylic acid metabolism \\
\hline Colnelenic acid & HMDB30996 & C16320 & 0.688 & alpha-Linolenic acid metabolism \\
\hline Traumatic acid & HMDB00933 & C16308 & 0.665 & alpha-Linolenic acid metabolism \\
\hline Stearidonic acid & HMDB06547 & C16300 & -0.965724523 & alpha-Linolenic acid metabolism \\
\hline Caffeyl alcohol & METPA1708 & C12206 & 0.689 & Phenylpropanoid biosynthesis \\
\hline Phytosphingosine & HMDB04610 & $\mathrm{C} 12144$ & -0.608 & Sphingolipid metabolism \\
\hline Abietinal & HMDB34735 & C11887 & -0.680 & Diterpenoid biosynthesis \\
\hline Picolinic acid & HMDB02243 & C10164 & -1.009229646 & Tryptophan metabolism \\
\hline Palmitoleic acid & HMDB03229 & C08362 & -1.552 & Fatty acid biosynthesis \\
\hline Alpha-Linolenic acid & HMDB01388 & C06427 & 0.672 & alpha-Linolenic acid metabolism \\
\hline $\begin{array}{l}\text { Guanosine } 2^{\prime}, 3^{\prime} \text {-cyclic } \\
\text { phosphate }\end{array}$ & HMDB11629 & C06194 & 1.852 & Purine metabolism \\
\hline Hydroxyphenylacetylglycine & HMDB00735 & C05596 & 2.027 & Tyrosine metabolism \\
\hline Galactosylglycerol & HMDB06790 & C05401 & 1.183 & $\begin{array}{l}\text { Galactose metabolism } \\
\text { Glycerolipid metabolism }\end{array}$ \\
\hline 2-Methoxyestradiol & HMDB00405 & C05302 & -0.991682395 & alpha-Linolenic acid metabolism \\
\hline 13(S)-HPOT & METPA0543 & C04785 & -1.989038788 & alpha-Linolenic acid metabolism \\
\hline $\begin{array}{l}\text { Imidazoleacetic acid } \\
\text { ribotide }\end{array}$ & HMDB06032 & C04437 & 0.681 & Histidine metabolism \\
\hline 5-Methylthioribose & HMDB01087 & C03089 & 1.626 & Cysteine and methionine metabolism \\
\hline Pimelic acid & HMDB00857 & C02656 & 0.753 & Biotin metabolism \\
\hline Capric acid & HMDB00511 & C01571 & 0.835 & Fatty acid biosynthesis \\
\hline Syringin & METPA1704 & C01533 & 0.968 & Phenylpropanoid biosynthesis \\
\hline 7,8-Diaminononanoate & METPA0113 & C01037 & -0.961929908 & Biotin metabolism \\
\hline Porphobilinogen & HMDB00245 & C00931 & 2.258 & Porphyrin and chlorophyll metabolism \\
\hline Sphinganine & HMDB00269 & C00836 & -1.600936161 & Sphingolipid metabolism \\
\hline LPA(0:0/18:2(9Z,12Z)) & HMDB07852 & C00416 & -0.704 & $\begin{array}{l}\text { Glycerolipid metabolism } \\
\text { Glycerophospholipid metabolism }\end{array}$ \\
\hline Guanosine & HMDB00133 & C00387 & 1.841 & Purine metabolism \\
\hline Xanthine & HMDB00292 & C00385 & 2.484 & Purine metabolism \\
\hline Geranyl-PP & HMDB01285 & C00341 & 0.759 & Terpenoid backbone biosynthesis \\
\hline Indoleacrylic acid & HMDB00734 & C00331 & 2.158484941 & Tryptophan metabolism \\
\hline Palmitic acid & HMDB00220 & C00249 & -1.552 & $\begin{array}{l}\text { Fatty acid biosynthesis } \\
\text { Fatty acid degradation } \\
\text { Cutin, suberine and wax biosynthesis } \\
\text { Fatty acid metabolism }\end{array}$ \\
\hline L-Valine & HMDB00883 & C00183 & -1.016522341 & $\begin{array}{l}\text { 2-Oxocarboxylic acid metabolism } \\
\text { Biosynthesis of amino acids } \\
\text { Valine, leucine and isoleucine } \\
\text { degradation } \\
\text { Valine, leucine and isoleucine } \\
\text { biosynthesis } \\
\text { Pantothenate and CoA biosynthesis } \\
\text { Aminoacyl-tRNA biosynthesis }\end{array}$ \\
\hline Proline & HMDB00162 & C00148 & -1.551840689 & $\begin{array}{l}\text { Biosynthesis of amino acids } \\
\text { Aminoacyl-tRNA biosynthesis } \\
\text { Arginine and proline metabolism }\end{array}$ \\
\hline L-Histidine & HMDB00177 & C00135 & -0.993 & Histidine metabolism \\
\hline
\end{tabular}


Table 1 Differentially expressed metabolites mapped to KEGG metabolic pathways (Continued)

\begin{tabular}{|c|c|c|c|c|}
\hline Metabolite Name & HMDB ID & $\begin{array}{l}\text { KEGGCompound } \\
\text { ID }\end{array}$ & $\begin{array}{l}\text { Fold change (sus_24 } \\
\text { vs.res_24) }\end{array}$ & Pathway Name \\
\hline & & & & $\begin{array}{l}\text { Aminoacyl-tRNA biosynthesis } \\
\text { Biosynthesis of amino acids }\end{array}$ \\
\hline L-Leucine & HMDB00687 & C00123 & -2.034 & $\begin{array}{l}\text { 2-Oxocarboxylic acid metabolism } \\
\text { Biosynthesis of amino acids } \\
\text { Valine, leucine and isoleucine } \\
\text { degradation } \\
\text { Valine, leucine and isoleucine } \\
\text { biosynthesis } \\
\text { Aminoacyl-tRNA biosynthesis }\end{array}$ \\
\hline Anthranilic acid & HMDB01123 & C00108 & 1.346 & $\begin{array}{l}\text { Biosynthesis of amino acids } \\
\text { Tryptophan metabolism } \\
\text { Phenylalanine, tyrosine and tryptophan } \\
\text { biosynthesis }\end{array}$ \\
\hline Phenylalanine & HMDB00159 & C00079 & -1.004301822 & $\begin{array}{l}\text { Phenylalanine metabolismPhenylalanine, } \\
\text { tyrosine and tryptophan biosynthesis } \\
\text { Phenylpropanoid biosynthesis } \\
\text { Tropane, piperidine and pyridine alkaloid } \\
\text { biosynthesis } \\
\text { Aminoacyl-tRNA biosynthesis } \\
\text { 2-Oxocarboxylic acid metabolism } \\
\text { Biosynthesis of amino acids }\end{array}$ \\
\hline Pyridoxal 5'-phosphate & HMDB01491 & C00018 & 1.153 & $\begin{array}{l}\text { Thiamine metabolism } \\
\text { Vitamin B6 metabolism }\end{array}$ \\
\hline
\end{tabular}

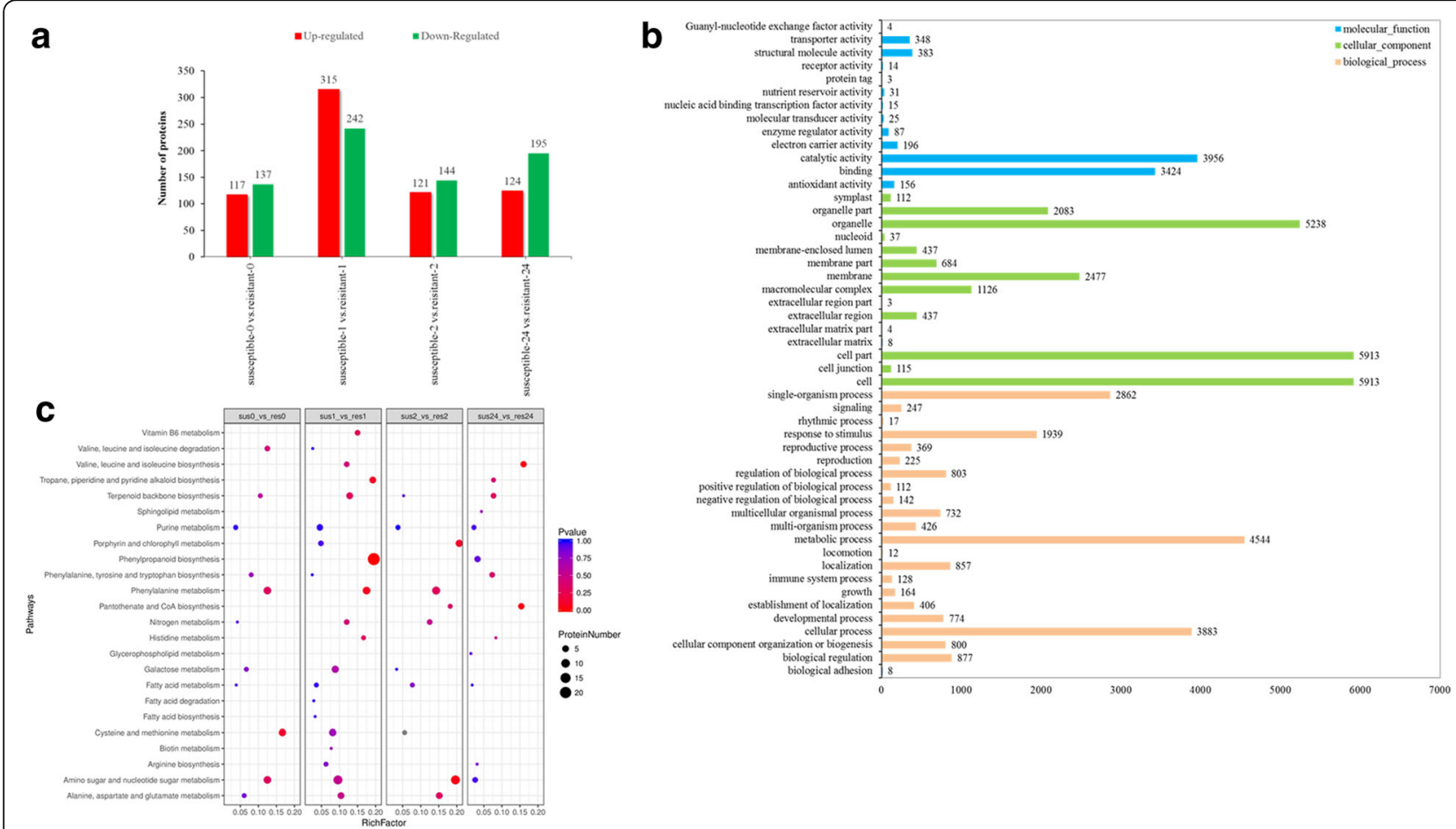

Fig. 2 Comparison of upregulated and downregulated proteins in resistant and susceptible genotypes (a); Differentially expressed proteins (DEPs) were identified by a Gene Ontology (GO) analysis (b); DEPs involved in metabolic pathways related to metabolites identified by LC-MS were dramatically affected by drought in annual ryegrass $(0,1,2$, and $24 \mathrm{~h})(\mathbf{c})$ 
low-quality Illumina HiSeq sequencing reads, approximately 137,708 unigenes were assembled. These annotated unigenes were used to search various functional databases, and unigenes that encoded transcription factors (TFs) were classified into different TF families (Additional file 5: Figure S4). Using a fold change $>2$ and FDR $<0.05$ as thresholds, transcripts from both resistant and susceptible genotypes were identified as DEGs. Many of the upregulated and downregulated DEGs that were highly expressed following drought treatment in the two L. multiflorum genotypes at different time points (Additional file 6: Figure S5), were enriched in various core metabolic pathways. Interestingly, a large number of DEGs that were involved in the pathway terms "sphingolipid metabolism," "pantothenate and CoA biosynthesis," "histidine metabolism," "glycerolipid metabolism," and "galactose metabolism," were observed in both L. multiflorum genotypes before drought treatment (Fig. 3a). In comparison to the drought-susceptible genotype, the expression of many DEGs were significantly increased in tolerant genotype after $1 \mathrm{~h}$ of drought, including those associated with "nitrogen metabolism," "cysteine and methionine metabolism," "citric acid cycle (TCA cycle)," "biotin metabolism," "arginine and proline metabolism," and "2-oxocarboxylic acid metabolism" (Fig. 3b). Similarly, more than 100 genes involved in "nitrogen metabolism," "cysteine and methionine metabolism," "biotin metabolism," and "biosynthesis of amino acids" were significantly enriched in tolerant genotype subjected to drought for $2 \mathrm{~h}$ (Fig. 3c). The number of DEGs involved in "tryptophan metabolism," "purine metabolism," "nitrogen metabolism," and "cutin, suberine, and wax biosynthesis" was increased in tolerant genotype, compared to sensitive genotype after $24 \mathrm{~h}$ of drought stress (Fig. 3d). These results indicate that the number of genes involved in these core metabolic processes showed significant changes.

To further confirm the RNA-Seq data, qRT-PCR was performed. The expression trends of 26 amino acid and lipid metabolism-related genes were consistent with RNA-Seq data in the two L. multiflorum genotypes

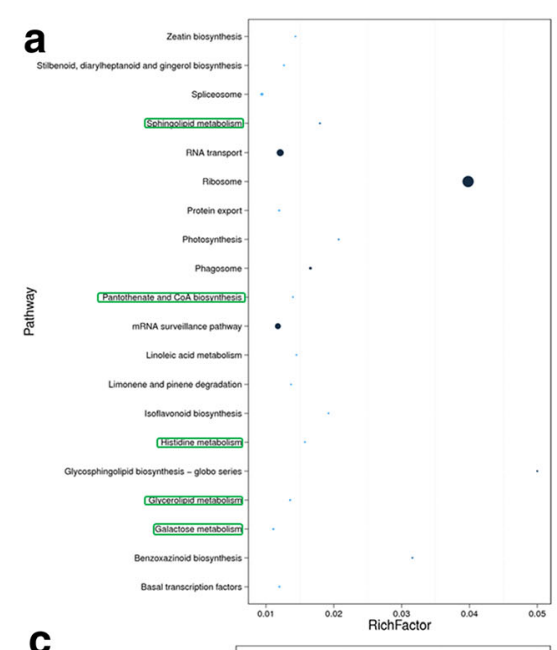

C

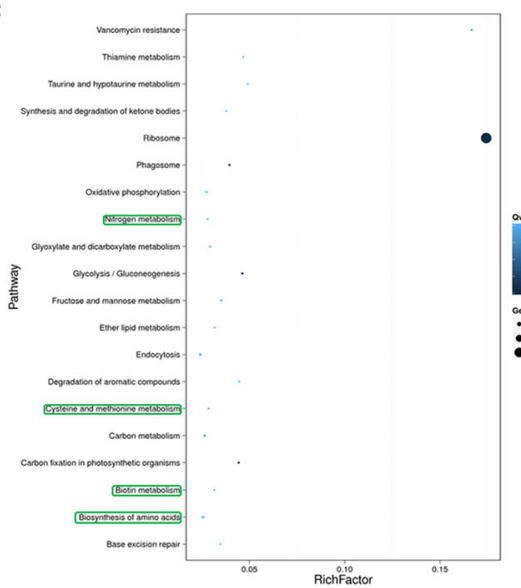

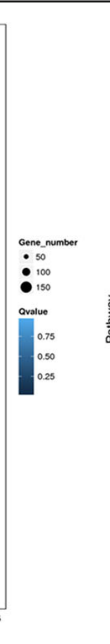

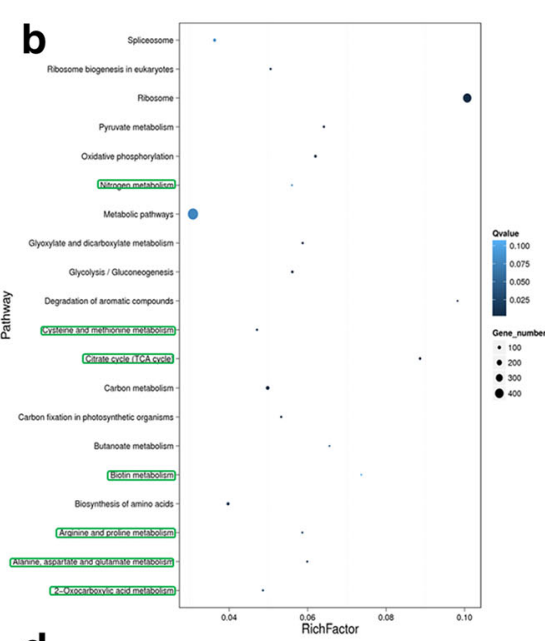

d
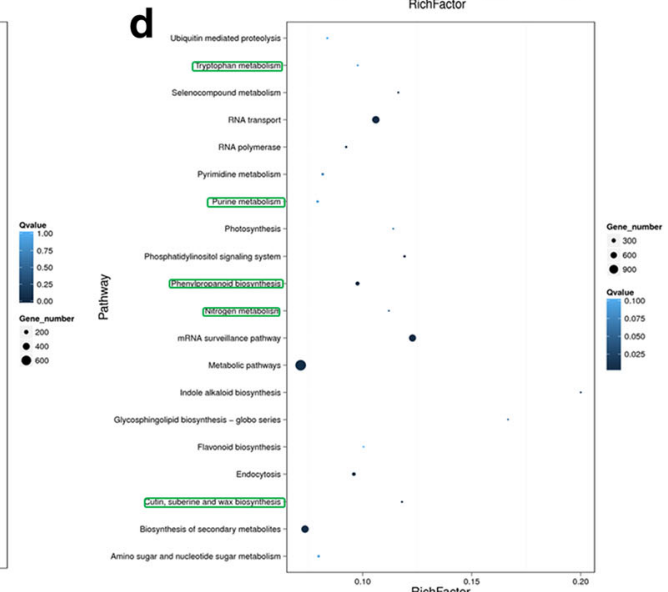

Fig. 3 Differentially expressed genes involved in core metabolic pathways in two L.multiflorum genotypes at four time points (0, 1, 2, and 24 h) during drought treatment $(\mathbf{a}-\mathbf{d})$ 
(Additional file 7: Figure S6), and fold changes in gene expression were significantly correlated in both the resistant and susceptible genotypes $\left(r^{2}>0.9\right)$.

\section{Multiple co-inertia analysis to evaluate the integration of omics datasets}

To demonstrate the relationships among transcripts, proteins, and metabolites, we collapsed our samples into control and drought-treated seedlings using the means of each omics profile. A strong correlation between the four datasets was observed (Fig. 4a). The top two MCoA components, responsible for more than $80 \%$ of the total variation (Fig. $4 \mathrm{~b}$ ), clearly separated the drought stress sensitive and resistant genotypes (Fig. 4a) The first and second components accounted for roughly comparable proportions of the variation, implying that both genetic background and drought treatment underlie considerable variation in the data.
As in the PCA, in which the contributions of variables (e.g., transcript, protein, and metabolite levels) to a component could have been evaluated by their loading vectors, variables with either positive or negative associations (i.e., with high absolute values) had significant influence on the co-inertia components. In contrast to the PCA, the variables comprising MCoA loading vectors were derived from all four datasets. In consideration of the biological meaning of the MCoA components, we subjected the loading vectors of the two components to GSEA. Pre-ranked analysis was performed, in which variables were ranked according to their loadings. The loading vectors for component 1 were significantly and positively associated with amino acid metabolism and lipid metabolism $(P<0.001$; Fig. $4 \mathrm{c}-\mathrm{d})$. Taken together, the omics data sets subjected to MCoA indicated that amino acid and lipid metabolism play important roles in annual ryegrass drought response.

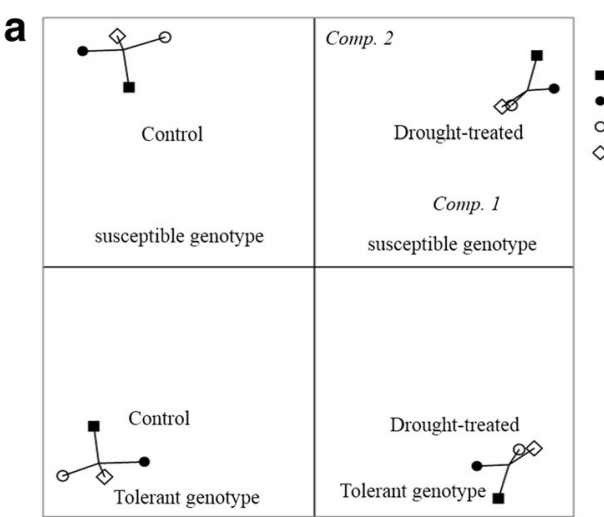

- mRNA

- Protein

Metabolite (negative)

$\diamond$ Metabolite (positive)

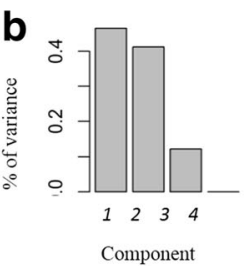

C

Pre-ranked loading vector 1

d Pre-ranked loading vector 1
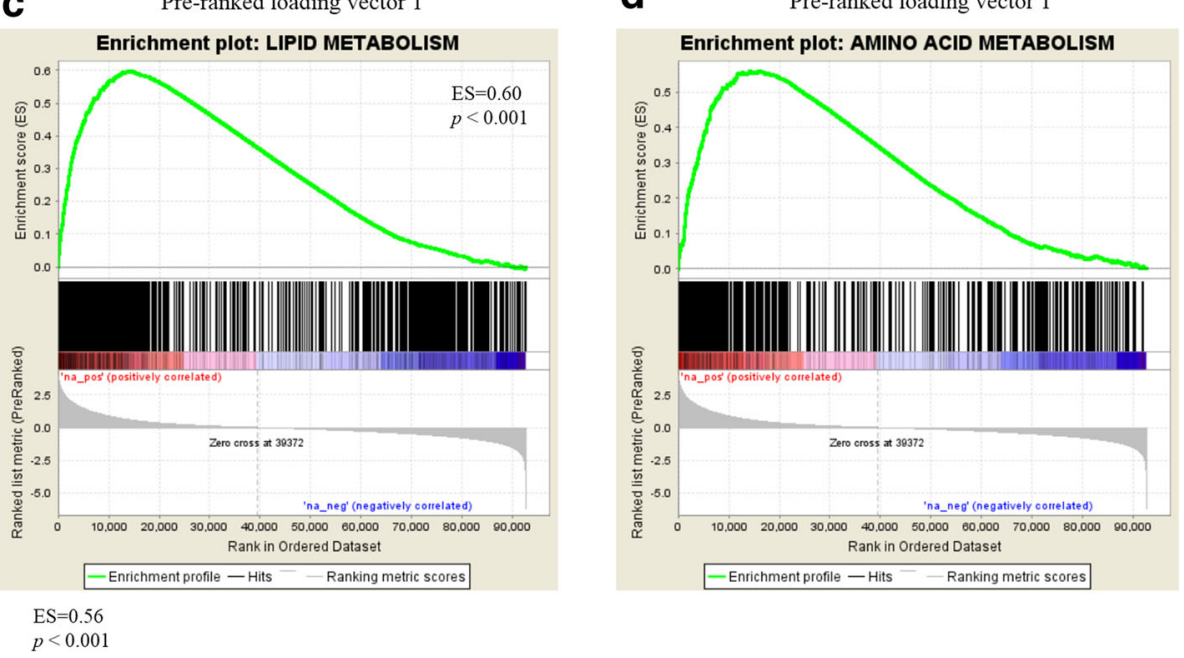

Fig. 4 Integrative analysis of multiple omics data using MCoA and GSEA. The first two components defined by MCoA (a). The proportion of variation explained by each component (b). The GSEA of the first loading vector for the lipid metabolism (c) and amino acid metabolism gene sets $(\mathbf{d})$. Each dot represents a plant genotype; the same genotype from different datasets are linked by segments. The length of segments connecting annual ryegrass is correlated with the similarity among datasets 
Targeted analysis revealed a significant association among transcripts, proteins, and metabolites in core metabolic processes

Correlation analysis was performed among differentially expressed metabolites, proteins, and genes (Fig. 5). We first matched all transcripts with their detected proteins, and an intermediate Pearson correlation coefficient of $r=0.724$ was observed. The DEGs with corresponding DEPs were classified into different groups exhibiting upregulation or downregulation. A total of 47 DEGs exhibited trends that matched those of their corresponding proteins in the two annual ryegrass genotypes subjected to drought stress. These down-regulated DEG and DEP pairs were involved in "phenylalanine, tyrosine, and tryptophan biosynthesis," "tropane, piperidine, and pyridine alkaloid biosynthesis," "phenylalanine metabolism," "phenylpropanoid biosynthesis," "biosynthesis of amino acids," "2-oxocarboxylic acid metabolism," and "glycerolipid metabolism." Similarly, 51 DEGs had contrasting expression changes with their corresponding proteins under drought stress, including genes involved in "valine, leucine, and isoleucine degradation" and "biosynthesis of amino acids." In addition, a large number of transcripts or proteins that had no significant regulation were also observed in core metabolic pathways.

Protein-gene-metabolite correlation networks were generated to examine responses of the 23 metabolic pathways in drought-resistant annual ryegrass (Table 2; Fig. 6). Integrated metabolic pathways were used to observe changes in enzymes, corresponding to levels of amino acids, lipids, carbohydrate conjugates, nucleosides, alkaloids and their derivatives, and pyridines and their derivatives. Several amino acids, such as L-valine, L-leucine, phenylalanine, L-histidine, and proline, were found in lower concentrations in the droughtsusceptible genotype than in the tolerant genotype under drought conditions. This may be a consequence of the downregulated key enzymes, including branched-chain amino acid aminotransferase (EC 2.6.1.42), tyrosine aminotransferase (EC 2.6.1.5), 5-methylthioribose kinase (EC 2.7.1.100), ATP phosphoribosyl transferase (EC

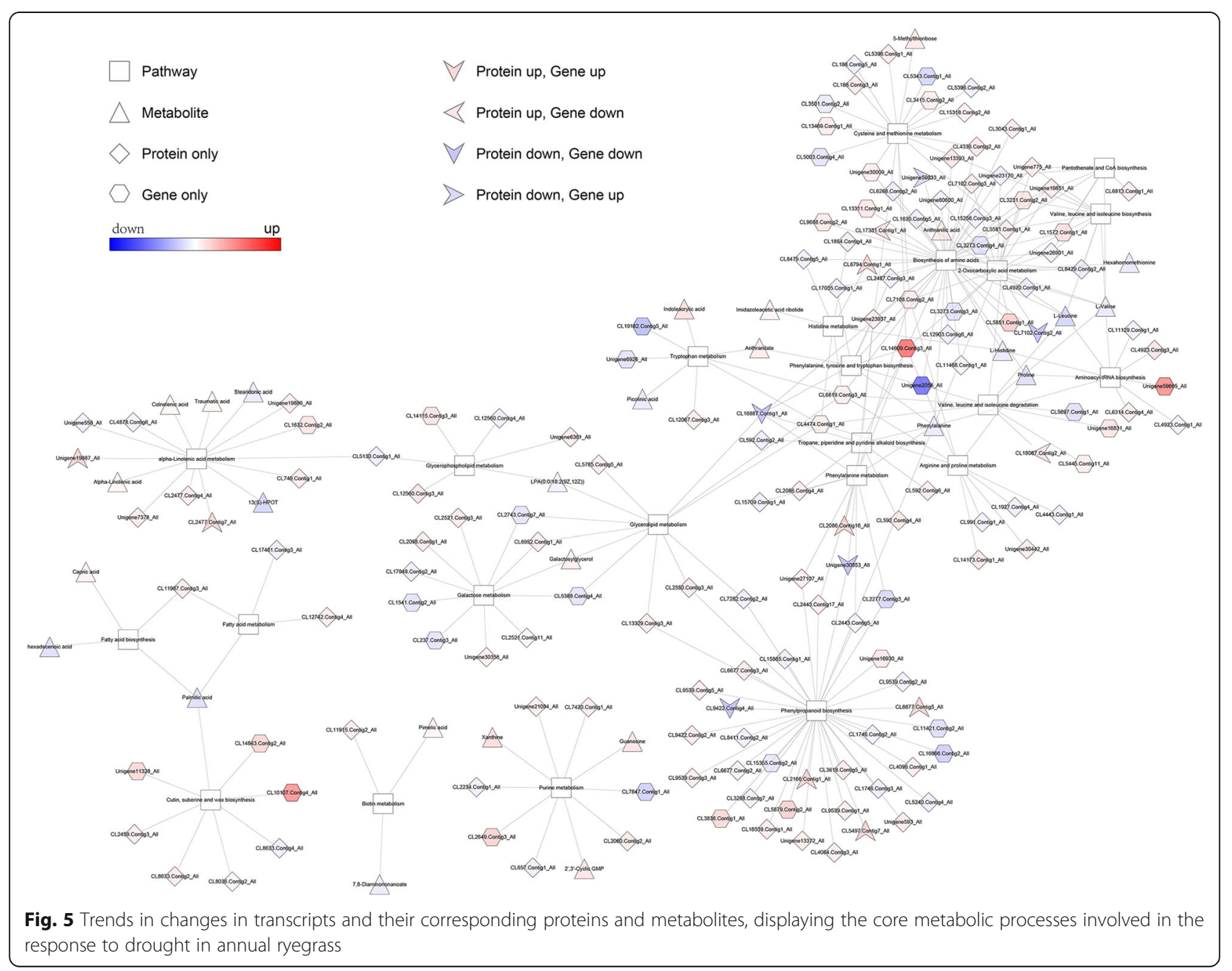


Table 2 Differentially expressed proteins and transcripts related to the core metabolism after drought in resistant and susceptible genotypes

\begin{tabular}{|c|c|c|c|}
\hline Access ion & Description, EC number & Protein ratio (sus_vs.res_) & Gene ratio (sus_vs.res_) \\
\hline Unigene26901_All & acetolactate synthase I/II/III large subunit [EC:2.2.1.6] & +1.27 & NA \\
\hline Unigene775_All & acetolactate synthase I/III small subunit [EC:2.2.1.6] & +1.35 & NA \\
\hline Unigene23170_All & branched-chain amino acid aminotransferase [EC:2.6.1.42] & -0.81 & NA \\
\hline Unigene775_All & acetolactate synthase I/III small subunit [EC:2.2.1.6] & +1.35 & NA \\
\hline CL2443.Contig5_All & phenylalanine/tyrosine ammonia-lyase [EC:4.3.1.25] & -0.54 & NA \\
\hline CL16887.Contig1_All & tyrosine aminotransferase [EC:2.6.1.5] & -0.77 & -0.77 \\
\hline CL2086.Contig4_All & primary-amine oxidase [EC:1.4.3.21] & +1.28 & NA \\
\hline CL2086.Contig16_All & primary-amine oxidase [EC:1.4.3.21] & +1.28 & +9.18 \\
\hline CL15709.Contig1_All & phenylpyruvatetautomerase [EC:5.3.2.1] & -0.77 & NA \\
\hline CL991.Contig1_All & agmatinedeiminase [EC:3.5.3.12] & -0.77 & NA \\
\hline CL17035.Contig1_All & tryptophan synthase alpha chain [EC:4.2.1.20] & -0.81 & NA \\
\hline CL12067.Contig3_All & L-tryptophan—pyruvate aminotransferase [EC:2.6.1.99] & +1.23 & NA \\
\hline Unigene30442_All & prolyl 4-hydroxylase [EC:1.14.11.2] & -0.82 & NA \\
\hline CL14173.Contig1_All & prolyl 4-hydroxylase [EC:1.14.11.2] & -0.74 & NA \\
\hline CL2487.Contig3_All & anthranilate synthase component I [EC:4.1.3.27] & -0.81 & NA \\
\hline CL4443.Contig1_All & ornithine decarboxylase [EC:4.1.1.17] & +1.79 & NA \\
\hline CL6314.Contig4_All & aspartyl-tRNAsynthetase [EC:6.1.1.12] & +1.24 & NA \\
\hline CL11129.Contig1_All & alanyl-tRNAsynthetase [EC:6.1.1.7] & -0.69 & NA \\
\hline CL6813.Contig1_All & dihydropyrimidinase [EC:3.5.2.2] & 1.22 & NA \\
\hline CL4336.Contig2_All & serine O-acetyltransferase [EC:2.3.1.30] & +1.6 & NA \\
\hline CL5396.Contig1_All & 5-methylthioribose kinase [EC:2.7.1.100] & +1.39 & NA \\
\hline CL5396.Contig2_All & 5-methylthioribose kinase [EC:2.7.1.100] & +1.39 & NA \\
\hline CL6794.Contig1_All & histidinol-phosphatase [EC:3.1.3.15] & +1.23 & NA \\
\hline CL1927.Contig4_All & Polyamine oxidase [EC: 1.5 .3 .14 1.5.3.16 1.5.3.] & -0.79 & NA \\
\hline CL11987.Contig3_All & acetyl-CoA carboxylase[EC:6.4.1.2] & +1.34 & NA \\
\hline CL8633.Contig2_All & omega-hydroxypalmitate O-feruloyltransferase [EC:2.3.1.188] & +1.33 & NA \\
\hline CL8036.Contig2_All & fatty acid omega-hydroxylase [EC:1.14.-.- ] & -0.5 & NA \\
\hline CL1854.Contig4_All & phosphoribosyl-AMP cyclohydrolase [EC 3.5.4.19] & -0.69 & NA \\
\hline CL1854.Contig4_All & phosphoribosyl-ATP pyrophosphohydrolase [EC:3.6.1.31] & -0.69 & NA \\
\hline CL8479.Contig5_All & ATP phosphoribosyltransferase [EC:2.4.2.17] & -0.68 & NA \\
\hline CL6952.Contig1_All & alpha-galactosidase [EC:3.2.1.22] & +1.23 & NA \\
\hline CL5785.Contig5_All & glycerol kinase [EC:2.7.1.30] & +1.27 & NA \\
\hline CL7163.Contig2_All & phosphatidatecytidylyltransferase [EC:2.7.7.41] & +1.23 & NA \\
\hline Unigene6361_All & phosphatidylserine decarboxylase [EC:4.1.1.65] & +1.32 & NA \\
\hline CL12560.Contig3_All & glycerophosphoryldiesterphosphodiesterase [EC:3.1.4.46] & +1.8 & NA \\
\hline CL2234.Contig1_All & glucan endo-1,3-beta-glucosidase 1/2/3 [EC:3.2.1.39] & -0.71 & NA \\
\hline CL7420.Contig1_All & AMP deaminase [EC:3.5.4.6] & +1.25 & NA \\
\hline Unigene21084_All & nucleoside-diphosphate kinase [EC:2.7.4.6] & +1.21 & NA \\
\hline Unigene19886_All & lipoxygenase [EC:1.13.11.12] & +1.24 & NA \\
\hline Unigene19887_All & lipoxygenase [EC:1.13.11.12] & +1.51 & NA \\
\hline CL2477.Contig4_All & lipoxygenase [EC:1.13.11.12] & +1.38 & NA \\
\hline CL2477.Contig7_All & lipoxygenase [EC:1.13.11.12] & +1.64 & NA \\
\hline CL749.Contig1_All & alcohol dehydrogenase class-P [EC:1.1.1.1] & +1.28 & NA \\
\hline
\end{tabular}


Table 2 Differentially expressed proteins and transcripts related to the core metabolism after drought in resistant and susceptible genotypes (Continued)

\begin{tabular}{llll}
\hline Access ion & Description, EC number & Protein ratio (sus_vs.res_) & Gene ratio (sus_vs.res_) \\
\hline CL17848.Contig2_All & hexokinase [EC:2.7.1.1] & -0.8 & NA \\
CL2098.Contig1_All & alpha-glucosidase [EC:3.2.1.20] & +1.46 & NA \\
CL2521.Contig11_All & beta-fructofuranosidase [EC:3.2.1.26] & +0.75 & NA \\
CL2521.Contig3_All & beta-fructofuranosidase [EC:3.2.1.26] & +1.3 & $\mathrm{NA}$ \\
Unigene30358_All & beta-fructofuranosidase [EC:3.2.1.26] & +1.3 & $\mathrm{NA}$ \\
Unigene7378_All & 12-oxophytodienoic acid reductase [EC:1.3.1.42] & +1.29 & $\mathrm{NA}$ \\
CL5130.Contig1_All & phospholipase A1 [EC:3.1.1.32] & -0.82 & $\mathrm{NA}$ \\
Unigene556_All & 12-oxophytodienoic acid reductase [EC:1.3.1.42] & -0.7 & $\mathrm{NA}$
\end{tabular}

2.4.2.17), phosphoribosyl-AMP cyclohydrolase (EC 3.5.4.19), phosphoribosyl-ATP pyrophosphohydrolase (EC 3.6.1.31), phenylpyruvate tautomerase (EC 5.3.2.1), agmatine deiminase (EC 3.5.3.12), prolyl 4-hydroxylase (EC 1.14.11.2), and the tryptophan synthase alpha chain (EC 4.2.1.20).

The increased levels of histidinol-phosphatase (EC 3.1.3.15) and primary-amine oxidase (EC 1.4.3.21) corresponded with the observed lower histone and phenylalanine concentrations. Similarly, a high level of serine $O$-acetyltransferase (EC 2.3.1.30) also corresponded with patterns in cysteine and serine metabolism. In addition, high levels of uracil conversion to $\beta$ alanine might have been supported by enhanced activity of dihydropyrimidinase (EC 3.5.2.2).

Expression of L-tryptophan-pyruvate aminotransferase (EC 2.6.1.99) is responsible for the formation of tryptophan from indolepyruvate, which is then converted into

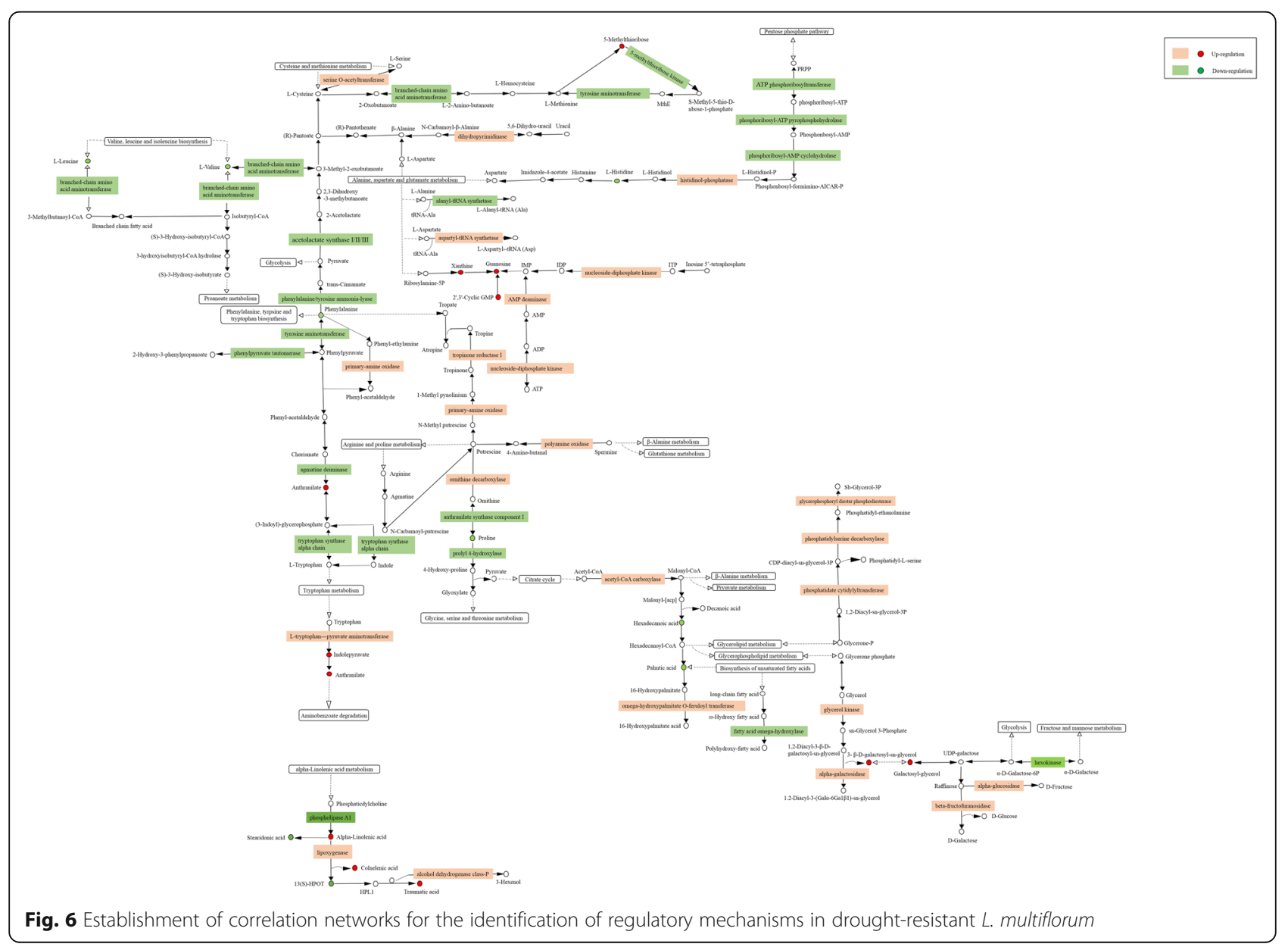


anthranilate. The ATP and AMP are directly converted to ADP and IMP via upregulation of nucleosidediphosphate kinase (EC 2.7.4.6) and AMP deaminase (EC 3.5.4.6), which could result in increased levels of xanthine, guanosine, and 2', 3'-cyclic GMP. Interestingly, acetyl-CoA carboxylase (EC 6.4.1.2) plays a vital role in increasing or reducing concentrations of hexadecenoic acid, capric acid, and palmitic acid. In resistant genotype, galactosylglycerol was significantly accumulated because of increased concentrations of glycerol kinase (EC 2.7.1.30) and reductions in the hexokinase pool.

In addition, the higher metabolic levels of alphagalactosidase (EC 3.2.1.22), alpha-glucosidase (EC 3.2.1.20), and beta-fructofuranosidase (EC 3.2.1.26) indicated stronger metabolism of galactose. Moreover, colnelenic acid, traumatic acid, stearidonic acid, alphalinolenic acid, and 13(S)-HPOT (9Z,11E,15Z)-(13S)-13Hydroperoxyoctadeca-9,11,15-trienoic acid) are all involved in alpha-linolenic acid metabolism, in which genes encoding enzymes, such as phospholipase A1 (EC 3.1.1.32), lipoxygenase (EC 1.13.11.12), and alcohol dehydrogenase class-P (EC 1.1.1.1) were each either strongly downregulated or upregulated.

\section{Discussion}

Plants subjected to water stress undergo a range of physiological changes [30]. In our previous study, in comparison to susceptible genotype, a higher level of CAT activity was exhibited in resistant genotype against short-term drought; however, SOD and MDA activity showed no significant differences between the two $L$. multiflorum genotypes when treated with $1 \mathrm{~h}$ of drought [20]. In contrast, in the present study, a significant difference was noted between the two annual ryegrass genotypes in response to long-term drought (Fig. 1). As the number of days of drought increased, tolerant plants exhibited higher levels of CAT and SOD activity, higher RWC and chlorophyll content, and lower MDA content (Fig. 1). Research conducted by Mastalerczuk et al. [31] and Borawska-Jarmujłowicz et al. [32] on perennial ryegrass showed that significant reductions in chlorophyll contents were observed after subjecting seedlings to abiotic stresses. These findings are consistent with those of the present study. Thus, the activity of antioxidant enzymes and changes in chlorophyll content play important roles in increasing the tolerance of resistant genotype to drought stress.

Alterations in membrane lipid composition represent an important response to environmental stresses $[33,34]$. Drought stress reduces the accumulation of fatty acids, such as palmitic acid, hexadecenoic acid, picolinic acid, and stearidonic acid [4, 35, 36], while substantially increasing the levels of most fatty acids, with the exception of pimelic acid, capric acid, (2’'E,4'Z,7'Z,8E)-colnelenic acid, alpha-linolenic acid, traumatic acid (TA) and acetyl-CoA carboxylase (ACCase) [37]. Tayeh et al. [38] showed that phospholipase A1 (PLA1) in plants plays important roles in the hydrolysis of phospholipids during cold stress. Andrade et al. [39] found that lipoxygenase (LOX) is involved in defense against drought stress in rice and sugarcane transgenic plants. Uma and Podile [40] reported that the transcripts encoding 9-LOX and a 9-LOX-derived compound, colnelenic acid, are significantly upregulated in resistant host tomato plants. Similarly, Cao et al. [41] reported that levels of alpha-linolenic acid and colnelenic acid were increased following treatment with exogenous methyl jasmonate (MeJA) . In the present study, phospholipase A1 enhanced the production of alpha-linolenic acid, which is converted to colnelenic acid; an effect that was directly induced by lipoxygenase in the tolerant annual ryegrass subjected to drought conditions (Fig. 6). These results showed that alpha-linolenic acid and colnelenic acid are involved in the regulation of membrane composition in drought-tolerant genotype.

In addition, TA is a plant wound hormone that can eliminate the negative effects of salt stress and oxidative stress in Chlorella vulgaris [42, 43]. Consistent with our previous results, a high TA content was detected in the resistant plants, indicating that the metabolite contributes to enhanced stress tolerance in drought-treated annual ryegrass. Moreover, levels of acetyl-CoA carboxylase (ACCase) and omega-hydroxypalmitate $\mathrm{O}$ feruloyl transferase in the annual ryegrass plants were enhanced by drought treatment. We found that reductions in the levels of palmitic acid and hexadecenoic acid were controlled by ACCase. The changes observed in ACCase and palmitic acid in lipid metabolism were consistent with the results of Kwan et al. [44].

Most free amino acids accumulate under drought stress [45], and this is thought to be an important adaptation [46, 47]. In the present study, downregulated branched-chain aminotransferase (BCAT) directly reduced L-valine and L-leucine levels. Anthranilate synthase (AS) causes a leucine-to-proline change, which leads to a reduction in the proline content of annual ryegrass. In addition, the upregulation of histidinolphosphatase is involved in L-histidine degradation. Furthermore, tyrosine amino transferase and phenylpyruvate tautomerase might be jointly involved in the degradation of phenylalanine. This finding has not been observed in previous studies.

The response of 5-methylthioribose to drought stress in drought-tolerant annual ryegrass is induced 
by cysteine and methionine metabolism, which was controlled in the present study by the two downregulated enzymes, tyrosine aminotransferase and 5methylthioribose kinase. Moreover, this compound has not been associated with drought tolerance in previous studies. Anthranilate acts as a precursor for tryptophan biosynthesis and reduces indoleacetic acid (IAA) synthesis [48]. Anthranilate formation resulted in indirect induction of IAA production, a novel finding in annual ryegrass that was consistent with the results of Hartmann and Zimmer [49].

The behavior of $2^{\prime}, 3^{\prime}$-cyclic nucleotide variants has been characterized in animal tissues, but not in plant materials [50]. According to our research, only $2^{\prime}, 3^{\prime}$-cyclic GMP was detected in annual ryegrass, which is involved in guanine production. Glucosylglycerol, found in many cyanobacteria, and galactosylglycerols (floridoside and isofloridoside) accumulate in eukaryotic algae under salt stress conditions [51]. In the present study, galactosylglycerol production was involved in glycolysis and fructose/mannose metabolism in response to drought, indicating that the concentration of galactosylglycerol plays a role in drought tolerance of annual ryegrass. In another study, galactose levels in drought-resistant potato genotypes were observed to be much higher than those in corresponding controls [52], a finding that is consistent with our results.

Transcription factors involved in transcriptional and post-transcriptional mechanisms regulate the accumulation of various metabolites by activating the expression of biosynthetic enzymes. Based on our omics data, various TF families, including MYB, bZIP, and bHLH, might regulate core metabolic processes during drought stress. The R2R3-MYB transcription factor AtMYB41 is induced in response to desiccation, and activates cuticle biosynthesis In Arabidopsis thaliana under biotic stress conditions [53]. In addition, AtMYB96 positively regulates the expression of lipidtransfer protein 3 (LTP3) via direct binding to the LTP3 promoter, and thereby enhances plant drought stress tolerance [54].

In response to drought stress, many plant species accumulate high levels of compatible osmolytes, such as Pro (proline), Gly betaine, or sugar alcohols, which are thought to be associated with stress adaptation. In higher plants, L-Pro is synthesized from L-Glu via delta1-pyrroline-5-carboxylate (P5C) by two enzymes, P5C synthetase (P5CS) and $\mathrm{P} 5 \mathrm{C}$ reductase (P5CR) under drought stress. The L-Pro is metabolized to L-Glu via P5C by two enzymes, Pro dehydrogenase (ProDH) and P5C dehydrogenase, during recovery from stress [55, 56].

Satoh and colleagues [57] first identified the group of S bZIP TFs, such as AtbZIP11, AtbZIP44, AtbZIP2, and AtbZIP53, which become involved in Pro metabolism by binding to the cis-acting element ACTCAT in the ProDH promoter. Later, Hanson and colleagues [58] reported that AtbZIP11 could affect amino acid metabolism by regulating the expression of ASPARAGINE SYNTHETASE1 and ProDH2 during low energy stress. Post-transcriptional regulatory mechanisms are also very important for drought stress tolerance in plants. It has been reported that AtbZIP1 and AtbZIP53 can be specifically heterodimerized with group $\mathrm{C}$ bZIP TFs to bind directly to the promoters of $A S$ PARAGINE SYNTHETASE1 and ProDH2 [59]. In addition, the Arabidopsis type-B response regulator 18 (ARR18) physically interacts with AtbZIP63; an interaction that negatively interferes with the transcriptional activity of AtbZIP63 on the ProDH1 promoter under osmotic stress [60]. Recently, the key TF AtbHLH112 involved in Pro metabolism was identified by Liu et al. [61]. AtbHLH112 could activate the expression of P5CS and repress the expression of $\mathrm{P} 5 \mathrm{CDH}$ and ProDH to increase Pro levels under abiotic stress tolerance. Taken together, the omics data suggest that post-transcriptional and transcriptional regulation of TFs that affect proteins and enzymes could mediate lipid and amino acid metabolism, thereby promoting adaptation to drought stress in annual ryegrass.

Over the last few decades, extensive efforts have been made to produce drought-tolerant ryegrass genotypes using molecular and biotechnological methods, such as the production of genetically modified or transgenic plants, with a focus on lipid and amino acid metabolism [62, 63]. Transgenic expression of some drought stress-related genes involved in lipid and amino acid metabolism could provide a more rapid strategy to achieve improved drought stress tolerance in ryegrass.

\section{Conclusions}

In order to explore the molecular mechanism associated with drought tolerance in two annual ryegrass genotypes, we identified differentially expressed metabolites and their corresponding proteins and transcripts that are involved in 23 core metabolic processes, in response to short-term drought stress. The regulatory networks were inferred using MCoA and correlation analysis to reveal the relationships among the expression of transcripts, proteins, and metabolites that highlight the corresponding elements of these core metabolic pathways. This study provides useful insights into the molecular mechanisms of drought resistance and represents a promising approach toward enhancing drought tolerance in forage grass. 


\section{Additional files}

Additional file 1: Figure S1. Sampling strategy for annual ryegrass under drought treatment for transcriptome, proteome, and metabolome analyses. (TIFF $2865 \mathrm{~kb}$ )

Additional file 2: Table S1. Detailed information of genes used for real-time-PCR analysis (DOCX $15 \mathrm{~kb}$ )

Additional file 3: Figure S2. Hierarchical clustering of different types of compounds detected in two L. multiflorum genotypes in the positive mode (A) and negative mode (B). (TIFF $1337 \mathrm{~kb}$ )

Additional file 4: Figure S3. Heatmaps of metabolite-metabolite correlations in positive mode (A) and negative mode (B). Metabolites were grouped by compound class, and each square represents the correlation between the metabolites indicated in the column and row headings. (TIFF $2818 \mathrm{~kb}$ )

Additional file 5: Figure S4. Summary of annotated unigenes identified using different functional databases (A) and unigenes that encode transcription factors (TFs) classified into TF families (B). (TIFF 1059 $\mathrm{kb})$

Additional file 6: Figure S5. Volcano plot of DEGs induced by drought treatment in two L. multiflorum genotypes at four time points. (TIFF 992 $\mathrm{kb})$

Additional file 7: Figure S6. The real-time PCR confirmation of RNASeq data in the drought-resistant (A) and drought-susceptible (B) annual ryegrass; Western blot validation of iTRAQ results in two L. multiflorum genotypes (C). (TIFF $2571 \mathrm{~kb}$ )

\section{Abbreviations}

ACT: Acetonitrile; ANOVA: Analysis of variance; APX: Ascorbic acid peroxidase; AS: Anthranilate synthase; CAT: Catalase; COG: Clusters of orthologous groups; DEG: Differentially expressed genes; DEM: Differentially expressed metabolites; DEP: differentially expressed proteins; FA: Formic acid; FDR: False discovery rate; FPKM: Fragments per kilobase of transcript sequence per million base pairs; GO: Gene Ontology; GSEA: Gene set enrichment analysis; IAA: Indoleacetic acid; IMP: Inosine monophosphate; KEGG: Kyoto Encyclopedia of Genes and Genomes; LOX: Lipoxygenase; LSD: Least significant difference; MCoA: Multiple co-inertia analysis; MDA: Malondialdehyde; MeJA: Methyl jasmonate; MGF: Mascot generic format; OPLS-DA: Orthogonal projections to latent structures discriminant analysis; PCA: Principal component analyses; PLS-DA: Partial least squares discriminant analysis; PVDF: Polyvinylidene fluoride; REC: Relative electrical conductivity; RP: Resolving power; RSR: Root-to-shoot ratio; RT: Retention time; RWC: Relative water content; SH: Seedling height; SOD: Superoxide dismutase; TA: Traumatic acid; TCA: Tricarboxylic acid; TF: Transcription factors; VIP: Variable importance in the project

\section{Acknowledgments}

Not applicable.

\section{Ethics approval and consent of participate}

The experimental research on plants (including seeds) performed in this study complies with institutional, national and international guidelines.

\section{Funding}

This research work was funded by the earmarked fund for Modern Agroindustry Technology Research System (No.CARS-35-05), the National Basic Research Program (973 program) in China (No 2014CB138705) and the National Natural Science Foundation of China (NSFC 31372363). All these funding play roles in the design of the study and collection, analysis, and in writing the manuscript.

\section{Availability of data and materials}

All the data pertaining to the present study has been included in table and/ or figure form in the manuscript and authors are pleased to share analyzed/ raw data and plant materials upon reasonable request; Raw Illumina reads are available in NCBI GEO: GSE78738 (https://www.ncbi.nlm.nih.gov). Other datasets supporting the conclusions of this article are included within the article and its additional files.

\section{Authors' contributions}

$L P, M Z$ and $X Z$ conceived and designed the experiments. ZY performed the sample collection and contributed to the data analyses. LP, CM and XF analyzed the data and drafted the manuscript. XM and JW revised and edited the text. All authors have read and approved the final manuscript.

\section{Consent for publication}

Not applicable.

\section{Competing interests}

The authors declare that they have no competing interests.

\section{Publisher's Note}

Springer Nature remains neutral with regard to jurisdictional claims in published maps and institutional affiliations.

\section{Author details}

'Department of Grassland Science, College of Animal Science and Technology, Sichuan Agricultural University, Chengdu, China. ${ }^{2}$ Chair of Proteomics and Bioanalytics, Technical University of Munich, 85354 Freising, Germany. ${ }^{3}$ Agronomy Department, University of Florida, Gainesville, USA. ${ }^{4}$ Vazyme Biotech Co., Ltd, Nanjing State Economy \& Technology Development Zone, Red Maple Technology Industrial Park, Nanjing, China. ${ }^{5}$ Institute of Crop Sciences, Chinese Academy of Agricultural Sciences, Beijing, China.

Received: 3 July 2017 Accepted: 17 January 2018

Published online: 30 January 2018

\section{References}

1. Borawska-Jarmułowicz B, Mastalerczuk G, Gozdowski D, Małuszyńska E, Szydłowska A. The sensitivity of Lolium Perenne and Poa Pratensis to salinity and drought during the seed germination and under different photoperiod conditions. Zemdirbyste-Agriculture. 2017:104(1):71-8.

2. Puranik S, Jha S, Srivastava PS, Sreenivasulu N, Prasad M. Comparative transcriptome analysis of contrasting foxtail millet cultivars in response to short-term salinity stress. J Plant Physiol. 2011;168(3):280-7.

3. Da Silva JV, Naylor AW, Kramer PJ. Some ultrastructural and enzymatic effects of water stress in cotton (Gossypium Hirsutum L.) leaves. P Natl Acad Sci. 1974;71(8):3243-7.

4. Bettaieb I, Zakhama N, Wannes WA, Kchouk M, Marzouk B. Water deficit effects on Salvia Officinalis fatty acids and essential oils composition. Sci Hortic Amsterdam. 2009:120(2):271-5.

5. Toumi I, Gargouri M, Nouairi I, Moschou P, Salem-Fnayou AB, Mliki A, Zarrouk M, Ghorbel A. Water stress induced changes in the leaf lipid composition of four grapevine genotypes with different drought tolerance. Biol Plant. 2008:52(1):161-4.

6. Zhou X, Jenks MA, Liu J, Liu A, Zhang X, Xiang J, Zou J, Peng Y, Chen X. Overexpression of transcription factor OsWR2 regulates wax and cutin biosynthesis in rice and enhances its tolerance to water deficit. Plant Mol Biol Rep. 2014;32(3):719-31.

7. Arbona V, Hossain Z, López-Climent MF, Pérez-Clemente RM, GómezCadenas A. Antioxidant enzymatic activity is linked to waterlogging stress tolerance in citrus. Physiol Plant. 2008;132(4):452-66.

8. Wang N, Zhao J, He X, Sun H, Zhang G, Wu F. Comparative proteomic analysis of drought tolerance in the two contrasting Tibetan wild genotypes and cultivated genotype. BMC Genomics. 2015;16(1):1.

9. Osorio S, Alba R, Damasceno CM, Lopez-Casado G, Lohse M, Zanor Ml, Tohge T, Usadel B, Rose JK, Fei Z. Systems biology of tomato fruit development: combined transcript, protein, and metabolite analysis of tomato transcription factor (nor, rin) and ethylene receptor (Nr) mutants reveals novel regulatory interactions. Plant Physiol. 2011;157(1):405-25.

10. Ricroch $A E$, Bergé $J B$, Kuntz $M$. Evaluation of genetically engineered crops using transcriptomic, proteomic, and metabolomic profiling techniques. Plant Physiol. 2011;155(4):1752-61.

11. Meng C, Zeleznik OA, Thallinger GG, Kuster B, Gholami AM, Culhane AC. Dimension reduction techniques for the integrative analysis of multi-omics data. Brief Bioinform. 2016;17(4):628-41.

12. Shi H, Jiang C, Ye T, D-x T, Reiter RJ, Zhang H, Liu R, Chan Z. Comparative physiological, metabolomic, and transcriptomic analyses reveal mechanisms 
of improved abiotic stress resistance in bermudagrass [Cynodon dactylon ( $L$ ). Pers.] by exogenous melatonin. J Exp Bot. 2015;66(3):681-94.

13. Decourcelle M, Perez-Fons L, Baulande S, Steiger S, Couvelard L, Hem S, Zhu C, Capell T, Christou P, Fraser P. Combined transcript, proteome, and metabolite analysis of transgenic maize seeds engineered for enhanced carotenoid synthesis reveals pleotropic effects in core metabolism. JExp Bot. 2015;66(11):3141-50.

14. Talukder S, Azhaguvel P, Mukherjee S, Young C, Tang Y, Krom N, Saha M. De novo assembly and characterization of tall fescue transcriptome under water stress. The Plant Genome. 2015;8(2):1-13. https://doi.org/10.3835/ plantgenome2014.09.0050.

15. Shi H, Ye T, Chan Z. Comparative proteomic responses of two bermudagrass (Cynodon Dactylon (L). Pers.) varieties contrasting in drought stress resistance. Plant Physiol Biochem. 2014;82:218-28.

16. Li M, Sheng G, Wu Y, Yu Z, Bañuelos GS, Yu H. Enhancement of nitrogen and phosphorus removal from eutrophic water by economic plant annual ryegrass (Lolium Multiflorum) with ion implantation. Environ Sci Pollut R. 2014;21(16):9617-25.

17. Yuan X, Ma Z, Pan M, Shi C. Microwave remote sensing of short-term droughts during crop growing seasons. Geophys Res Lett. 2015:42(11): 4394-401.

18. Antolin M, Yoller J, Sanchez-Diaz M. Effects of temporary drought on nitrate-fed and nitrogen-fixing alfalfa plants. Plant Sci. 1995;107(2):159-65.

19. Perlikowski D, Kosmala A, Rapacz M, Kościelniak J, Pawłowicz I, Zwierzykowski Z. Influence of short-term drought conditions and subsequent re-watering on the physiology and proteome of Lolium Multiflorum/Festuca Arundinacea introgression forms, with contrasting levels of tolerance to long-term drought. Plant Biol. 2014;16(2):385-94.

20. Pan L, Zhang X, Wang J, Ma X, Zhou M, Huang L, Nie G, Wang P, Yang $Z$, Li J. Transcriptional profiles of drought-related genes in modulating metabolic processes and antioxidant defenses in Lolium Multiflorum. Plant Sci. 2016;7:519.

21. Barrs $\mathrm{H}$, Kozlowski T. Determination of water deficits in plant tissues. Water Deficits and Plant Growth. 1968;1:235-368.

22. Lakra N, Nutan KK, Das P, Anwar K, Singlapareek SL, Pareek A. A nuclearlocalized histone-gene binding protein from rice (OsHBP1b) functions in salinity and drought stress tolerance by maintaining chlorophyll content and improving the antioxidant machinery. J Plant Physiol. 2015;176:36-46.

23. Medeiros JS, Pockman WT. Drought increases freezing tolerance of both leaves and xylem of Larrea Tridentata. Plant Cell Environ. 2011;34(1):43-51.

24. Kanehisa M, Goto S. KEGG: Kyoto encyclopedia of genes and genomes. Nucleic Acids Res. 2000;28:27-30

25. Kanehisa M, Furumichi M, Tanabe M, Sato Y, Morishima K. KEGG: new perspectives on genomes, pathways, diseases and drugs. Nucleic Acids Res. 2017;45:353-61.

26. Kanehisa M, Sato Y, Kawashima M, Furumichi M, Tanabe M. KEGG as a reference resource for gene and protein annotation. Nucleic Acids Res. 2016;44:457-62.

27. Garg N, Kapono CA, Lim YW, Koyama N, Vermeij MJA, Conrad D, Rohwer F, Dorrestein PC. Mass spectral similarity for untargeted metabolomics data analysis of complex mixtures. Int J Mass Spectrom. 2014:377:719-27.

28. Meng C, Culhane A. Integrative exploratory analysis of two or more genomic datasets. Stat Genom. 2016;1418:19-38

29. Meierhofer D, Weidner C, Hartmann L, Mayr JA, Han C-T, Schroeder FC, Sauer S. Protein sets define disease states and predict in vivo effects of drug treatment. Mol Cell Proteomics. 2013;12(7):1965-79.

30. Bowne JB, Erwin TA, Juttner J, Schnurbusch $T$, Langridge $P$, Bacic $A$, Roessner $U$. Drought responses of leaf tissues from wheat cultivars of differing drought tolerance at the metabolite level. Mol Plant. 2012; 5(2):418-29.

31. Mastalerczuk G, Borawska-Jarmułowicz B, Kalaji HM, Dąbrowski P, Paderewski J. Gas-exchange parameters and morphological features of festulolium (Festulolium braunii K. Richert a. Camus) in response to nitrogen dosage. Photosynthetica. 2017;55(1):20-30.

32. Borawskajarmułowicz B, Mastalerczuk G, Pietkiewicz S, Kalaji MH. Low temperature and hardening effects on photosynthetic apparatus efficiency and survival of forage grass varieties. Plant Soil Environ. 2014;60(4):177-83.

33. Burgess $P$, Gianfagna T, Huang B. Fatty acid and metabolism in leaves and roots associated with improved drought tolerance in Agrostis Stolonifera expressing SAG12-IPT gene controlling cytokinin synthesis. Int Turf Res J. 2013;12:497-502.
34. Campo S, Baldrich P, Messeguer J, Lalanne E, Coca M, San Segundo B. Overexpression of a calcium-dependent protein kinase confers salt and drought tolerance in rice by preventing membrane lipid peroxidation. Plant Physiol. 2014;165(2):688-704.

35. Benhassaine Kesri G, Aid F, Demandre C, Kader JC, Mazliak P. Drought stress affects chloroplast lipid metabolism in rape (Brassica Napus) leaves. Physiol Plant. 2002;115(2):221-7.

36. Wenzel A, Frank T, Reichenberger G, Herz M. Impact of induced drought stress on the metabolite profiles of barley grain. Metabolomics. 2015;11(2):454-67.

37. Upchurch RG. Fatty acid unsaturation, mobilization, and regulation in the response of plants to stress. Biotechnol Lett. 2008;30(6):967-77.

38. Tayeh C, Randoux B, Laruelle F, Bourdon N, Reignault P. Lipids as markers of induced resistance in wheat: a biochemical and molecular approach. Commun Agric Appl Biol Sci. 2013;78(3):459-65.

39. Andrade LM, Benatti TR, Nobile PM, Goldman MH, Figueira A, Marin ALA, Brito MS, Silva J, Creste S. Characterization, isolation and cloning of sugarcane genes related to drought stress. BMC Proc. 2014:8(4):P110.

40. Uma B, Podile AR. Overlapping sets of transcripts from host and non-host interactions of tomato are expressed early during non-host resistance. Plant OMICS. 2014;7(1):19-27.

41. Cao J, Li M, Jian C, Pei L, Zhen L. Effects of MeJA on Arabidopsis metabolome under endogenous JA deficiency. Sci Rep. 2016;6:37674.

42. Pietryczuk A, Biziewska I, Imierska M. Influence of traumatic acid on growth and metabolism of Chlorella Vulgaris under conditions of salt stress. Plant Growth Regul. 2014;73(2):103-10.

43. Jabłońskatrypuć A, Pankiewicz W, Czerpak R. Traumatic acid reduces oxidative stress and enhances collagen biosynthesis in cultured human skin fibroblasts. Lipids. 2016:51(9):1021-35.

44. Kwan HY, Niu X, Dai W, Tong T, Chao X, Tao S, Chi LC, Lee KC, Fu X, Hua Y. Lipidomic-based investigation into the regulatory effect of Schisandrin B on palmitic acid level in non-alcoholic steatotic livers. Sci Rep. 2014;5:9114.

45. Rai VK. Role of amino acids in plant responses to stresses. Biol Plant. 2002; 45(4):481-7.

46. Holmstrup M, Slotsbo S, Rozsypal J, Henriksen PG, Bayley M. Accumulation of free amino acids during exposure to drought in three springtail species. J Insect Physiol. 2015;82:114-21.

47. Srivastava A, Sinha S. Uncoupling of ammonia channel as a mechanism of allosteric inhibition in anthranilate synthase of Serratia Marcescens: dynamic and graph theoretic analysis. Mol Biosyst. 2016;13(1):142-155. https://doi. org/10.1039/C6MB00646A.

48. Spaepen S, Vanderleyden J, Remans R. Indole-3-acetic acid in microbial and microorganism-plant signaling. FEMS Microbiol Rev. 2007;31(4):425-48.

49. Hartmann A, Zimmer W. Physiology of Azospirillum. Azospirillum/plant associations (Okon Y, ed). Boca Raton: CRC Press; 1994:15-39.

50. Van DT, Blancquaert D, Couturon P, Van DSD, Sandra P, Lynen F. Wounding stress causes rapid increase in concentration of the naturally occurring $2^{\prime}, 3^{\prime}-$ isomers of cyclic guanosine- and cyclic adenosine monophosphate (cGMP and (AMP) in plant tissues. Phytochemistry. 2014;103:59-66.

51. Hagemann M, Pade N. Heterosides-compatible solutes occurring in prokaryotic and eukaryotic phototrophs. Plant Biol. 2015;17(5):927-34.

52. Vasquezrobinet C, Mane SP, Ulanov AV, Watkinson JI, Stromberg VK, De KD, Schafleitner R, Willmot DB, Bonierbale M, Bohnert HJ. Physiological and molecular adaptations to drought in Andean potato genotypes. J Exp Bot. 2008;59(8):2109.

53. Cominelli E, Sala T, Calvi D, Gusmaroli G, Tonelli C. Over-expression of the Arabidopsis AtMYB41 gene alters cell expansion and leaf surface permeability. Plant J. 2008;53(1):53-64.

54. Lin $G$, Yang $H$, Zhang $X$, Yang S. Lipid transfer protein 3 as a target of MYB96 mediates freezing and drought stress in Arabidopsis. J Exp Bot. 2013;64(6):1755

55. Liu J, Zhu JK. Proline accumulation and salt-stress-induced gene expression in a salt-hypersensitive mutant of Arabidopsis. Plant Physiol. 1997;114(2):591-6.

56. Satoh R, Nakashima K, Seki M, Shinozaki K, Yamaguchishinozaki K. ACTCAT, a novel cis-acting element for proline- and hypoosmolarity-responsive expression of the ProDH gene encoding proline dehydrogenase in Arabidopsis. Plant Physiol. 2002;130(2):709.

57. Satoh R, Fujita Y, Nakashima K, Shinozaki K, Yamaguchi-Shinozaki K. A novel subgroup of bZIP proteins functions as transcriptional activators in hypoosmolarity-responsive expression of the ProDH gene in Arabidopsis. Plant Cell Physiol. 2004;45(3):309-17. 
58. Hanson J, Hanssen M, Wiese A, Hendriks MM, Smeekens S. The sucrose regulated transcription factor bZIP11 affects amino acid metabolism by regulating the expression of ASPARAGINE SYNTHETASE1 and PROLINE DEHYDROGENASE2. Plant J. 2008;53(6):935-49.

59. Dietrich K, Dröge-Laser W. Heterodimers of the Arabidopsis transcription factors bZIP1 and bZIP53 reprogram amino acid metabolism during low energy stress. Plant Cell. 2011;23(1):381.

60. Veerabagu M, Kirchler T, Elgass K, Stadelhofer B, Stahl M, Harter K, MiraRodado V, Chaban C. The interaction of the Arabidopsis response regulator ARR18 with bZIP63 mediates the regulation of PROLINE DEHYDROGENASE expression. Mol Plant. 2014;7(10):1560.

61. Liu Y, Ji X, Nie X, Qu M, Zheng L, Tan Z, Zhao H, Huo L, Liu S, Zhang B. Arabidopsis AtbHLH112 regulates the expression of genes involved in abiotic stress tolerance by binding to their E-box and GCG-box motifs. New Phytol. 2015:207(3):692-709.

62. Campo S, Baldrich P, Messeguer J, Lalanne E, Coca M, San SB.

Overexpression of a calcium-dependent protein kinase confers salt and drought tolerance in Rice by preventing membrane lipid peroxidation. Plant Physiol. 2014;165(2):688.

63. Lyon D, Castillejo MA, Mehmetitershani V, Staudinger C, Kleemaier C, Wienkoop S. Drought and recovery: independently regulated processes highlighting the importance of protein turnover dynamics and translational regulation in Medicago Truncatula. Mol Cell Proteomics. 2016;15(6):1921-37.

\section{Submit your next manuscript to BioMed Central} and we will help you at every step:

- We accept pre-submission inquiries

- Our selector tool helps you to find the most relevant journal

- We provide round the clock customer support

- Convenient online submission

- Thorough peer review

- Inclusion in PubMed and all major indexing services

- Maximum visibility for your research

Submit your manuscript at www.biomedcentral.com/submit 